\title{
Construcción y validación de una escala de sensibilidad a la crítica en un grupo de universitarios de Lima metropolitana
}

\author{
Ramón León \& Manuel Avellaneda*
}

La presente comunicación reporta la construcción y validación de una escala de evaluación de sensibilidad a la crítica, dimensión psicológica de gran importancia en la psicología clínica, la psicoterapia, la psicología educacional y la psicología organizacional, que sin embargo ha sido poco estudiada.

Los autores construyeron una relación de 20 itemes, que hacían referencia a la condición socioeconómica, las características psicológicas, el rendimiento académico y los rasgos conductuales y actitudinales de las personas, que se respondiera (en una escala Likert de 0-10) cuánto les afectaría una crítica sobre el particular por parte de su mejor amigo o amiga.

Sensibilidad a la crítica / validez de constructo / confiabilidad / análisis psicométrico.

\section{Development of a scale of sensitivity to criticism in a group of students of Lima, Peru}

The present communication informs about the construction and validation process of a psychometric reactive which evaluates the sensitivity to criticism, a psychological construct of inmense importance in the clinic, educational and organizational psychology.

The authors constructed a 20-items reactive with topics as the zone of residence, the social abilities, academic achievement, and some other characteristics, to be evaluated with a Likert-Scale (0 to 10) as a motive of critic by close friends. The subjects were asked to response to what extent were they hurt by this critic.

Sensitivity to criticism / construct validity / psychometric analysis.

Correo electrónico: rleon@correo.ulima.edu.pe

* Asistente de investigación. 
La sensibilidad a la crítica es una dimensión psicológica poco estudiada de modo sistemático, pero que fácilmente puede ser observada en la vida cotidiana. Todos conocemos personas que reaccionan ya sea de un modo airado o, por el contrario, con sentimientos de insuficiencia que pueden estar cercanos a la depresión, ante comentarios, observaciones y cuestionamientos formulados por otros.

Tal vez por eso esta dimensión, aunque no ha sido investigada y conceptuada de modo sistemático sino hasta hace poco tiempo, es mencionada (en algunos casos de manera incidental, en otros con cierto detenimiento) por numerosos autores a lo largo del siglo XX. Uno de ellos fue Alfred Adler, el llamado padre de la psicología individual, quien hace referencia a ella en El carácter neurótico (Adler, 1985). En las páginas de esta obra, Adler, primero discípulo y después disidente de Freud, describe la irritabilidad, el egocentrismo, la escasa tolerancia a la frustración y la mínima capacidad para aceptar la opinión divergente, todos estos rasgos propios de la personalidad neurótica, en la cual -según él- el sentimiento de inferioridad tiene un rol crucial.

Comentarios puntuales, rectificaciones, planteamientos alternativos o sencilla y llanamente opiniones divergentes, provocan en personalidades neuróticas reacciones emocionales muchas veces desmedidas, que van desde el desánimo manifiesto hasta la réplica agresiva.
Desde otro punto de vista teórico, Ernst Kretschmer, una de las grandes figuras de la psiquiatría en la centuria pasada, propuso lo que se conoce con el nombre de delirio sensitivo de referencia (Kretschmer, 1966). Bajo esta denominación se describe una particular forma de sensibilidad a eventuales comentarios negativos que desarrollan personas tímidas o de una autoestima endeble al creer que los demás perciben las insuficiencias de ellas o algunos de sus rasgos negativos.

Kurt Schneider, otra de las grandes figuras de la psiquiatría alemana, en Las personalidades psicopáticas (Schneider, 1968), destaca igualmente la presencia de sensibilidad a la crítica, por ejemplo entre las personalidades ansiosas de notoriedad ("necesitados de estimación", como él los llama). Al otro lado del Atlántico podemos mencionar a Harry Stack Sullivan, uno de los más perfilados representantes del neopsicoanálisis y figura de gran influencia en la psiquiatría norteamericana (Perry, 1982). Su obra, parva en la cantidad pero rica en observaciones y planteamientos teóricos, contiene numerosas referencias a la sensibilidad a la crítica. Así, en La teoría interpersonal de la psiquiatría, Sullivan describe lo que ocurre en la etapa escolar con los siguientes términos:

Algunos de esos niños son tratados con la mayor crudeza por otros de su misma edad. En esta etapa -aunque no sea más que por que el niño acaba de llegar de su hogar y su experiencia anterior ha sido siempre con personas mayores y herma- 
nos menores, o con compañeros imaginarios de juego- hay una insensibilidad verdaderamente horrible hacia los sentimientos de valor personal en las otras personas (Sullivan, 1964: 268).

Esas experiencias tempranas, que lastiman tanto física como psíquicamente, son caldo de cultivo para posteriores trastornos.

Por último, en la moderna clasificación del DSM-IV la marcada sensibilidad a la crítica aparece entre los rasgos definitorios de personalidades limítrofes y narcisistas (Millon, 2000), particularmente vulnerables a cualquier apreciación de los otros que ellas puedan interpretar como negativas. Ebert et al. (2000) destacan, de otro lado, la presencia de una percepción muy aguda entre personalidades con trastornos paranoides frente a supuestos ataques, críticas y postergaciones.

Algunos reactivos que exploran entidades diagnósticas propuestas en el DSM-IV incluyen ítems que evalúan la sensibilidad a la crítica; por ejemplo, la escala creada por Claridge \& Broks (1984) para evaluar la personalidad esquizotípica.

Estamos, pues, ante un rasgo de la personalidad que constituye un elemento decisivo para la estabilidad psicológica y para la autoimagen de las personas; se trata de un constructo de gran significación en un amplio espectro de dominios psicológicos (Atlas, 1994). Boyce et al. (1993) se refieren a él como un factor de gran importancia en la sensibi- lidad interpersonal, que supone además un marcado interés con respecto a las respuestas de la sociedad con respecto a lo que uno hace, la vigilancia de las reacciones de los otros, y una preocupación asimismo marcada acerca del comportamiento y afirmaciones de los otros. La Interpersonal Sensitivity Measure, que Boyce \& Parker (1989) desarrollaran, incluye también ítems que evalúan sensibilidad a la crítica ("if someone is critical of something I do, I feel bad", "I worry about being criticized for things that I have said or done", "I feel hurt when someone is angry with me", "I always expect criticism") ["si alguien critica algo que hago, me siento mal", "me preocupa ser critic.ado por cosas que he dicho o hecho", "me siento herido cuando alguien se enoja conmigo", "siempre espero ser criticado"]. (Harb et al., 2002).

Personas que padecen de fobia social tenderán a presentar marcada sensibilidad a la crítica o a percibir comentarios muchas veces incidentales como reproches que lastiman su autoestima (Hall \& Goldberg, 1977; Lucock \& Salkovskis, 1988; Snyder et al., 1985; Cimbrado, 2001). Entre personalidades depresivas también se puede verificar una excesiva sensibilidad a la crítica (Beck et al., 1979). Perugi et al. (2003) encontraron que depresivos atípicos ciclotímicos presentaban marcada sensibilidad interpersonal y patrones de evitamiento en las relaciones interpersonales. Hooley 
\& Teasdale (1989) señalan que este rasgo era un predictor de eventuales recaídas en cuadros depresivos.

No solo en el ámbito de lo psicopatológico la sensibilidad a la crítica es un constructo de importancia. También lo es en el de la educación y la industria, razón por la cual educadores y empresarios suelen prestarle gran atención al momento de considerar las formas en que deben manifestar a los alumnos o a los trabajadores su opinión acerca de su rendimiento. Baron (1988) ha señalado que la crítica destructiva suele resultar en mayores niveles de enojo, tensión y tendencias a la autodevaluación así como a disminuir el nivel de las metas que las personas se proponen. La crítica destructiva asume, por lo general, la forma de comentarios sarcásticos o excesivamente duros, desconsiderados en el tono, con contenidos amenazantes y atribuciones de los bajos rendimientos a causas internas, e implican una valoración más bien general que la precisión de aspectos deficitarios (Ilgen et al., 1981).

Por cierto, no siempre es fácil expresar opiniones o valoraciones negativas acerca del rendimiento de un subordinado o un colega, de modo tal que esto no sea percibido como una agresión. Es por eso que muchos gerentes reportan que dar a conocer apreciaciones negativas acerca de su rendimiento a sus subordinados es una de las tareas más difíciles y displacenteras (Larson, 1984, 1986; Veiga, 1988).
Snodgrass encontró, de otra parte, que los subordinados tienden a ser más impactados por las críticas de sus jefes que los jefes con respecto a las que les formulan sus subordinados (Snodgrass, 1992). Asimismo, se ha reportado que las mujeres son más sensibles a la crítica que los hombres (may, 1984; Wittig \& Skolnick, 1978). Wittkower \& Wittkower (2000) han tratado de la marcada vanidad y extrema sensibilidad a la crítica entre los artistas de la Antigüedad. Un dramático ejemplo de las devastadoras consecuencias de una sensibilidad aguda a una crítica prolongada y acerba se encuentra en el monólogo "Antes del desayuno", del dramaturgo norteamericano Eugene O'Neill (1960).

La crítica y la reacción a ella ocupan un lugar de central importancia en el mundo académico y científico. Todos conocemos las habituales secciones de "revisiones críticas" en revistas especializadas, en las cuales se comenta las cualidades y las deficiencias de libros, con la posibilidad de réplica por parte de los autores comentados.

La psicología misma ofrece excelentes ejemplos de la sensibilidad a la crítica de algunas de sus personalidades: pensemos en la reacción de Freud ante las formulaciones de Adler (Handlbauer, 1990), y en las duras controversias entre Wundt y Stumpf (Boring, 1978).

Cercano a la psicología, el mundo de la sociología nos ofrece asimismo un ejemplo casi paradigmático en el com- 
portamiento de Karl Marx con respecto a quienes se atrevían a disentir de sus puntos de vista. Johnson (2000) habla de su "irascibilidad extrema" en su trato con otros eruditos. Grunfeld (1980), citando a Schurz, es más preciso:

Trataba al que no estaba de acuerdo con él con un desprecio poco disimulado. Contestaba a todos los argumentos que le disgustaban con un desdén mordaz por la lastimosa ignorancia de los que se le acercaban... (p. 25).

Como siempre sucede con los conceptos psicológicos, uno de los mayores problemas es el referido a la operacionalización del término, tarea fundamental que precede al desarrollo de reactivos que posibiliten la evaluación. De más está decir que los conceptos psicológicos por lo general se muestran esquivos a la tarea operacionalizadora y que no siempre es fácil establecer criterios precisos y satisfactorios (Berrios, 2000).

En cuanto a la sensibilidad a la crítica, mucho del trabajo de investigación en las décadas de los setenta y ochenta se concentró en la sensibilidad a expresiones no verbales de emociones, utilizando para tal efecto fotografías o filmaciones (Buck, 1975; Ekman, 1973;), o en el autorreporte en inventarios de personalidad de elección forzada (Davis, 1980, 1983).

La crítica es una actividad exclusivamente humana. Criticar o ser criticado son circunstancias limitadas siempre a la especie humana. La crítica se expresa siempre en palabras y supone una valo- ración del comportamiento de los otros a la luz de parámetros normativos, y puede expresarse abiertamente o de manera solapada. En algunas sociedades, por ejemplo en la norteamericana, se puede constatar la presencia de una extendida cultura de la crítica, mientras que en otras, como la latinoamericana, la crítica no siempre es bienvenida o apenas esperada. Octavio Paz en México (Paz, 1990), y Julio Ortega en el Perú (Ortega, 1978), han anotado la escasa capacidad para formular críticas y la mínima tolerancia para recibirla en sus respectivas sociedades.

Como dijimos, la crítica puede expresarse de manera abierta o también de modo oculto y a espaldas del criticado. Cuando esto ocurre se suele hablar de maledicencia (gossip en inglés; Klatsch en alemán), un tema acerca del cual la bibliografía no es muy amplia (e.g. Spaks, 1985; Goodman \& BenZe`ev, 1994; Levin \& Arluke, 1987).

La maledicencia, que como su nombre lo indica tiene un fuerte componente malévolo y de hostilidad hacia quien es su víctima, es una señal de alerta y al mismo tiempo un mecanismo de control social, pues si ella llega a extenderse amenaza con convertirse en escándalo, es decir una situación en la cual el buen nombre de una persona es severamente puesto en tela de juicio con escasa capacidad por parte de ella para controlar los daños morales y materiales que aquel le pueda causar. 
Aunque existen algunos defensores de la maledicencia, como el gran poeta Auden (Auden, 1996; véase también Gooman \& Ben-Ze'ev, 1994), por lo general es condenada por todas las sociedades. Como siempre sucede, hay estereotipos vinculados a ella: Steiner (1995) habla del estereotipo de la anciana desdentada y de mirada maliciosa que vigila con ojos hipercríticos el comportamiento de los demás, en especial de las personas más jóvenes de su propio sexo.

La maledicencia es un fenómeno cotidiano. Ha existido en todos los tiempos, es tan antigua como la humanidad misma (Pérez, 1997), y si bien se la censura y prohíbe, lo cierto es que se la practica todos los días (Goodman \& Ben-Ze'ev, 1994) y en todas las culturas (Gluckman, 1963). "To tell me doesn't like gossip is like someone telling me he doesn't like Queen's Pudding. I simply cannot understand it, nor do I approve", ["Que me digan que no gusta el chisme es como que alguien me diga que no le gusta el Queen's Pudding. Simplemente no lo entiendo ni lo apruebo"], escribe Robert Morley, el conocido actor británico, en sus Morley Marvels (citado por Pepper, 1987).

Puede decirse que ella es el tratamiento detenido con otra persona de actos y características por lo general negativos e indicadores de algún tipo de deficiencia de una tercera persona. En Gossip, Patricia Meyer Spaks (1985) describe la maledicencia como "malicia destilada". "(La maledicencia) -escribe- juega con reputaciones, haciendo circular verdades y medias verdades y falsedades acerca de los actos y algunas veces sobre los motivos y sentimientos de los otros". Jane Caputi (1993), por su parte, señala que la maledicencia trata de lo que no se habla, identifica y expone a los protagonistas, dice verdades, satiriza y ridiculiza.

$\mathrm{Y}$, sin embargo, en alguna medida la maledicencia es una forma de filosofar. Danhauser, citado por Medini y Rosenberg (1976), señala que "la maledicencia no es nunca tan trivial como la gente piensa". En realidad, sostiene, "es la más cercana aproximación del hombre común a la filosofía" (p. 452). Por su parte, Forrester (1995) es del parecer de que el conocimiento obtenido por medio del chisme "describe más bien esa tierra de nadie que es la fantasía, pero que constituye igualmente el conocimiento social por el que vivimos" (p. 298). Desde una perspectiva más inmediata, el chisme puede ser visto como un conjunto de noticias informales acerca de otras personas (Levin \& Arluke, 1987; Stirling, 1956).

Ciertamente, el constructo de sensibilidad a la crítica no hace referencia solo al temor de ser objeto de comentarios maliciosos a espaldas de uno. Tiene que ver con el modo como las personas se sienten impactadas ante comentarios manifiestos acerca de sus patrones de reacción, actitudes, conductas, vestimenta, entorno familiar y social, rendimientos laborales y académicos. 
Es comprensible que en una sociedad como la actual, que enfatiza el individualismo, la competitividad, la asertividad y el éxito, personas particularmente sensibles a la crítica experimenten numerosas dificultades que se manifiestan en una serie de conductas carenciales, tales como temor a hablar en público, incapacidad para afirmarse, enrojecimiento del rostro y en general en una imagen social desvaída y opaca. En algunos casos estas características pueden llevar al desarrollo de ideas suicidas como lo señalan algunos autores.

Dada la singularidad del constructo, no se han desarrollado reactivos psicométricos que permitan evaluarlo con precisión. Algunos autores se han acercado a él de manera tangencial. Por ejemplo, Mehrabian (1976) desarrolló un inventario que evaluaba el rechazo social, concepto que guarda relación con la sensibilidad a la critica evidentemente. Ya hemos señalado, asimismo, que Boyce \& Parker (1989), al desarrollar su Interpersonal sensitivity measu$r e$, incluyen ítems que evalúan la sensibilidad a la crítica. Posteriormente, en 1994 Atlas publicó un artículo en el que informaba de la construcción de un inventario para evaluar de modo específico la sensibilidad a la crítica. $\mathrm{Su}$ reactivo, consistente en 24 ítems, evaluaba en primer lugar áreas de sensibilidad a la crítica y enseguida proponía una viñeta en la que un amigo le formula una crítica a la persona que responde el reactivo. Las propiedades psicométricas del reactivo creado por Atlas fueron adecuadas (Atlas, 1994).

No cabe duda de que el constructo de sensibilidad a la crítica tiene fuertes vínculos con los diversos contextos culturales. Existen conductas criticables en todas las culturas, como robar, matar o causar daño a una persona, pero dejando estas de lado, hay características y comportamientos que en algunas culturas $y$ épocas han sido objeto de crítica y en otras han sido aceptadas. Ver llorar a un niño no provoca rechazo, mientras que ocurre lo contrario cuando se trata de una persona del sexo masculino mayor.

Basándonos en estos razonamientos preparamos un reactivo que evalúa la sensibilidad a la crítica especialmente dirigido a estudiantes universitarios.

Objeto de la crítica pueden ser las más diversas características: desde aspectos tan concretos como la imagen corporal hasta dimensiones tan sutiles como los estilos de interacción social, pasando por dominios tales como la zona de residencia, las características del cónyuge o la elección de pareja.

Sin duda alguna, hay también variaciones de corte cultural e histórico que influyen en la crítica. Hasta hace algunas décadas la condición del homosexual era sometida a una severa crítica y hasta a la penalización, mientras que hoy la homosexualidad suele ser vista como una opción sexual y en la mayoría de los países ha sido despenalizada (Fone, 2000). 
De otro lado, en ciertas etapas de la vida algunos factores cobran una importancia mayor que otros. Así, por ejemplo, entre los jóvenes del mundo moderno los elementos vinculados a su imagen corporal, al empleo de ropa de moda y de marca, poseen un significado muy grande.

\section{MATERIAL y MÉTODO}

Para construir el instrumento se seleccionó un conjunto de 20 características corporales, sociales, psicológicas y de interrelación personal, a fin de proponerlas como eventuales aspectos de crítica. Entre ellos estaban la apariencia física, la capacidad económica, la zona de residencia, la universidad en la que estudia, la ropa y los pasatiempos.

En la selección se incluyeron factores como los de corte psicológico (por ejemplo, la presencia de determinados rasgos de personalidad considerados como deseables tales como la capacidad para generar simpatías, la capacidad para expresarse), así como también otros, más bien referidos a contextos socioculturales y económicos (zona de residencia, universidad en la que se estudia, etcétera).

A continuación se formularon instrucciones en las que se pedía a las personas que respondieran calificando sobre la base de una escala de Likert (0-10) cuánto les afectaría una crítica formulada por su mejor amigo o amiga.

En el anexo se presenta la versión final de la escala trabajada en la presente investigación.

\section{Participantes}

Un total de 517 estudiantes (189 varones y 328 mujeres), entre 17 y 31 años, que cursaban estudios en dos universidades privadas (Universidad Ricardo Palma y Universidad de Lima) y una universidad estatal (Universidad Nacional Federico Villarreal) respondieron anónima y colectivamente al reactivo antes descrito en el marco de una investigación más amplia que también evaluaba aspectos de autoasignación racial y de autoasignación en términos de clase social.

\section{Resultados}

La tabla 1 presenta las principales cualidades psicométricas del reactivo creado. Los resultados se refieren a la muestra total.

Los ítems 17 (tu familia), 7 (tu personalidad), 3 (tu rendimiento académico), y 20 (tu cultura general) obtuvieron los promedios más elevados; es decir, en caso de ser objeto de crítica serían experimentados como particularmente negativos. En el otro polo, el ítem 4 (tu distrito de residencia) obtuvo el promedio más bajo.

El análisis de la correlación ítemtotal de todos los ítems arrojó como resultado que todas las correlaciones estaban por encima de 0.60 , y el coeficiente Alfa de Cronbach fue igualmente muy elevado (.95), lo que indica que la escala es internamente consistente. 
Tabla 1

Análisis psicométrico de los ítemes de la Escala de Sensibilidad a la Crítica

\begin{tabular}{rrrr}
\hline Ítem & $\mathrm{M}$ & $\mathrm{D} . \mathrm{E}$. & $\mathrm{r}_{\text {itc }}$ \\
\hline 1 & 4.62 & 2.61 & $0.62^{*}$ \\
2 & 3.70 & 2.82 & $0.62^{*}$ \\
3 & 5.15 & 2.85 & $0.61^{*}$ \\
4 & 2.94 & 2.76 & $0.65^{*}$ \\
5 & 3.16 & 2.86 & $0.66^{*}$ \\
6 & 4.34 & 3.10 & $0.70^{*}$ \\
7 & 5.23 & 3.16 & $0.74^{*}$ \\
8 & 4.70 & 2.89 & $0.74^{*}$ \\
9 & 3.39 & 2.97 & $0.72^{*}$ \\
10 & 3.31 & 2.83 & $0.76^{*}$ \\
11 & 3.44 & 3.16 & $0.73^{*}$ \\
12 & 4.01 & 3.22 & $0.61^{*}$ \\
13 & 3.18 & 2.76 & $0.70^{*}$ \\
14 & 4.62 & 2.25 & $0.70^{*}$ \\
15 & 3.25 & 2.88 & $0.67^{*}$ \\
16 & 4.14 & 3.06 & $0.77^{*}$ \\
17 & 5.28 & 3.70 & 0.64 * \\
18 & 4.59 & 3.03 & 0.76 * \\
19 & 4.62 & 3.10 & $0.73^{*}$ \\
20 & 5.01 & 3.34 & $0.73^{*}$ \\
\hline \multicolumn{5}{c}{ Coeficiente Alfa de Cronbach $=.95$} \\
\hline *
\end{tabular}

${ }^{*} p<.05 ; \quad N=517$

Las características de la Escala de Sensibilidad a la Crítica permiten afirmar que ella posee validez y apropiada consistencia interna. La tabla 1 demuestra que la correlación ítem-total de todos los ítems va de 61 (ítems $3 \mathrm{y}$ 12, tu rendimiento académico y el enamorado que tienes, respectivamente) a .77 (ítem 16, tu capacidad para caer simpático). El coeficiente de Cronbach de toda la escala fue de .95 .

\section{Validación de constructo}

A continuación se procedió a llevar a cabo la validación de constructo del
Tabla 2

Análisis factorial exploratorio de la Escala de Sensibilidad a la Crítica

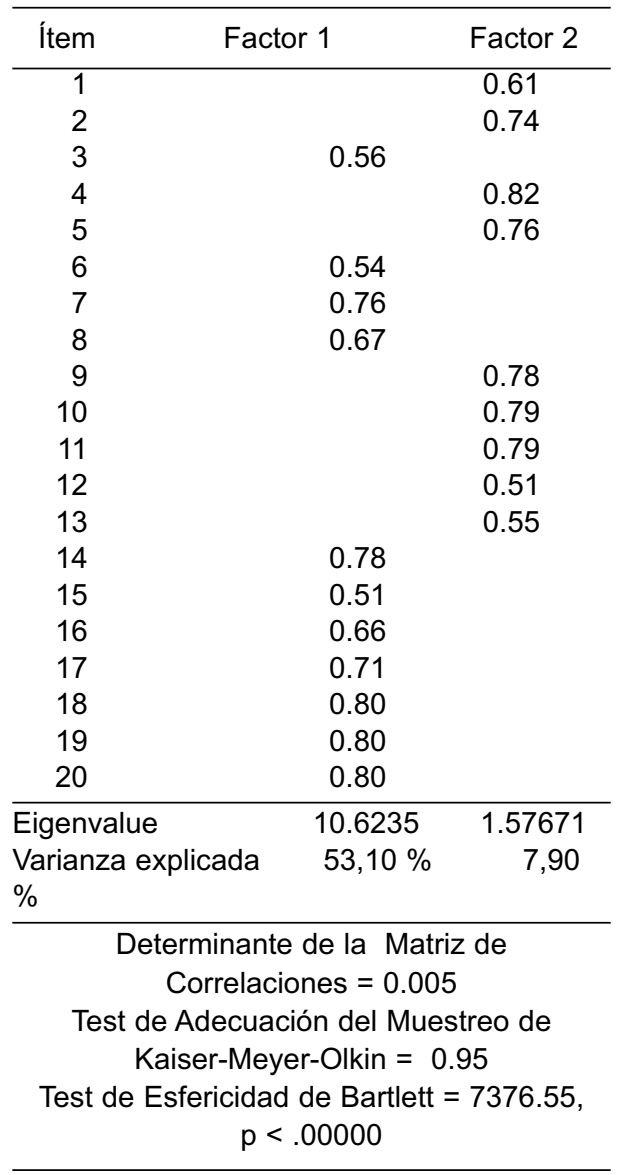

${ }^{*} p<.05 ; N=517$

reactivo propuesto. Para el efecto se procedió a realizar un análisis factorial exploratorio, a través del método de factorización de los componentes principales y rotación ortogonal Varimax. Los resultados se presentan en la tabla 2 .

Como puede observarse, se obtuvieron dos factores que explican el 61 por ciento de la varianza. El primero está 
conformado por ítems que describen las características que debe tener un líder, tales como ser aceptado por los demás, capacidad para tomar decisiones y habilidades sociales; es decir, rasgos de personalidad. El segundo agrupa ítems que tienen que ver con la imagen social, enfatizando los aspectos económicos, culturales y sociales; esto es, los aspectos de status.

El análisis psicométrico del primer factor de la Escala de Sensibilidad a la Crítica, presentado en la tabla 3, indica que la totalidad de las correlaciones ítem-total se encuentran por encima de .40 , lo que indica que todos los ítems se pueden considerar válidos. De otro lado, puede notarse que el coeficiente Alfa de Cronbach es elevado (0.93), por lo que concluimos que la escala presenta confiabilidad.

Tabla 3

Análisis psicométrico del Factor I, Rasgos de Personalidad, de la Escala de Sensibilidad a la Crítica

\begin{tabular}{cccc}
\hline Ítem & $\mathrm{M}$ & D.E. & $\mathrm{r}_{\text {itc }}$ \\
\hline 3 & 5.15 & 2.85 & 0.60 * \\
6 & 4.34 & 3.10 & 0.66 * \\
7 & 5.23 & 3.16 & 0.78 * \\
8 & 4.70 & 2.89 & 0.74 * \\
14 & 4.62 & 2.25 & $0.73^{*}$ \\
15 & 3.25 & 2.88 & 0.62 * \\
16 & 4.14 & 3.06 & $0.75^{*}$ \\
17 & 5.28 & 3.70 & 0.67 * \\
18 & 4.59 & 3.03 & 0.80 * \\
19 & 4.62 & 3.10 & 0.78 * \\
20 & 5.01 & 3.34 & 0.78 * \\
\hline
\end{tabular}

Coeficiente Alfa de Cronbach $=0.93$

${ }^{*} p<.05 ; \quad N=517$
El ítem con el promedio más elevado fue el 17, tu familia, y el que obtuvo el promedio más bajo fue el $15, t u$ rendimiento en algún deporte.

Tabla 4

Análisis psicométrico del Factor II, Imagen Social, de la Escala de Sensibilidad a la Crítica

\begin{tabular}{cccc}
\hline İtem & $\mathrm{M}$ & $\mathrm{D} . \mathrm{E}$. & $\mathrm{r}_{\text {itc }}$ \\
\hline 1 & 4.62 & 2.61 & $0.61^{*}$ \\
2 & 3.70 & 2.82 & $0.67^{*}$ \\
4 & 2.94 & 2.76 & 0.74 * \\
5 & 3.16 & 2.86 & $0.71^{*}$ \\
9 & 3.39 & 2.97 & $0.78^{*}$ \\
10 & 3.31 & 2.83 & 0.82 * \\
11 & 3.44 & 3.16 & $0.70^{*}$ \\
12 & 4.01 & 3.22 & 0.60 * \\
13 & 3.18 & 2.76 & $0.67^{*}$ \\
\hline
\end{tabular}

Coeficiente Alfa de Cronbach $=0.91$

${ }^{*} p<.05 ; \quad N=517$

Pasemos ahora a examinar el siguiente factor, cuyas propiedades psicométricas son presentadas en la tabla 4 , notándose que los ítems obtienen correlaciones elevadas $(0.60$ y superiores) con el puntaje total. El ítem 1 ( $t u$ apariencia física) fue el que obtuvo el promedio más elevado, y el 4 ( $t u$ distrito de residencia) el más bajo.

También se encuentra que el coeficiente Alfa de Cronbach es elevado (0.91), por lo que se puede concluir que la escala es confiable.

El análisis de las correlaciones entre los factores y el puntaje total, en la tabla 5, hizo posible observar que las dos escalas correlacionan de modo ele- 
Tabla 5

Correlaciones entre los factores y el total de la escala de Sensibilidad a la Crítica

\begin{tabular}{lcl}
\hline Variables & Puntaje Total & Factor 2 \\
\hline Factor 1 & $0.95^{* * *}$ & $0.95^{* * *}$ \\
Factor 2 & $0.94^{* * *}$ & \\
\hline
\end{tabular}

${ }^{* * *} \mathrm{p}<.001 ; \mathrm{N}=517$

vado entre sí y también con el puntaje total. Esto permite afirmar que ambas escalas evalúan dos aspectos complementarios de un mismo constructo.

La tabla 6 presenta los baremos de los puntajes promedio de los ítems obtenidos por rango percentil para cada uno de los factores y para la escala total. Con la finalidad de realizar una interpretación más apropiada, los puntajes corresponden a los promedios de los ítems y no a los de los puntajes totales de las subescalas y a la escala en su totalidad. Esto permite comparar directamente los puntajes obtenidos por cada sujeto en cada uno de los 20 ítems. Posteriores experiencias con este reactivo proporcionarán la base de datos para la elaboración de baremos de los puntajes totales.

\section{Discusión}

Dado que el estudio reportado tiene un carácter exploratorio, lo más que se puede decir es que la escala tiene utilidad con fines de investigación con adolescentes, pues no se ha ensayado este
Tabla 6

Baremos para la Escala de Sensibilidad a la Crítica

\begin{tabular}{ccccc}
\hline Percentil & Factor 1 & Factor 2 & Total & Percentil \\
\hline 01 & .00 & .00 & .00 & 01 \\
05 & .54 & .33 & .53 & 05 \\
10 & 1.18 & .66 & 1.15 & 10 \\
15 & 1.63 & 1.00 & 1.55 & 15 \\
20 & 2.18 & 1.33 & 1.99 & 20 \\
25 & 2.72 & 1.66 & 2.36 & 25 \\
30 & 3.18 & 2.04 & 2.69 & 30 \\
35 & 3.72 & 2.33 & 3.11 & 35 \\
40 & 4.09 & 2.77 & 3.54 & 40 \\
45 & 4.54 & 3.22 & 3.81 & 45 \\
50 & 4.81 & 3.44 & 4.15 & 50 \\
55 & 5.09 & 3.77 & 4.50 & 55 \\
60 & 5.45 & 4.00 & 4.81 & 60 \\
65 & 5.72 & 4.33 & 5.00 & 65 \\
70 & 6.18 & 4.66 & 5.24 & 70 \\
75 & 6.54 & 5.00 & 5.60 & 75 \\
80 & 6.81 & 5.37 & 5.94 & 80 \\
85 & 7.18 & 5.77 & 6.29 & 85 \\
90 & 7.27 & 6.57 & 6.92 & 90 \\
95 & 8.36 & 7.67 & 7.93 & 95 \\
99 & 9.34 & 9.22 & 9.00 & 99 \\
\hline $\mathrm{M}$ & 4.08 & 4.63 & 3.53 & $\mathrm{M}$ \\
$\mathrm{D} . \mathrm{E}$. & 2.18 & 2.42 & 2.22 & $\mathrm{D} . \mathrm{E}$ \\
\hline $\mathrm{N}$ & & 517 & & $\mathrm{~N}$ \\
& & & &
\end{tabular}

reactivo en contextos clínicos ni tampoco se tiene experiencias con ella en muestras de adultos. Sin embargo, las características psicométricas de la escala obtenidas a través de este estudio pueden ser consideradas como adecuadas y, por lo tanto, justifican estudios en muestras más amplias o de otras edades.

El ítem en el cual se halla el promedio más elevado (o sea, aquel que refleja que la crítica ha afectado a quien 
la recibe) es el 17, tu apariencia física, algo esperable ya que la etapa de la adolescencia se distingue, entre otras cosas, por una intensa preocupación precisamente por la apariencia física. Lo mismo puede afirmarse del ítem 7, tu personalidad, que obtiene el segundo promedio más alto.

Los dos ítems que siguen en cuanto a promedios más elevados, el 3 (tu rendimiento académico) y el 20 (tu cultura general) reflejan las preocupaciones de los integrantes de la muestra, todos universitarios.

El análisis factorial arrojó dos factores. El primero refleja las preocupaciones por la evaluación que los demás hacen de los rasgos de personalidad de uno. Esto, resulta obvio, ocurre en las personas a lo largo de toda su vida, pero se manifiesta en una intensidad más elevada entre adolescentes y jóvenes (Horrocks, 1984), como también en personas con predisposición a cuadros de depresión y a la ansiedad social, un fenómeno comórbido de la depresión (Harb et al., 2002).

La actual insistencia en poseer habilidades sociales como un elemento central en el desarrollo de una carrera profesional, así como para una vida de relación caracterizada por la actividad y la asertividad (Niven, 2003), debe haber contribuido a la presencia de este factor en una muestra como la que hemos empleado para el presente estudio, conformada por estudiantes universitarios.
Eso explica la presencia de los ítems en los cuales aspectos como la capacidad para hacer valer los derechos propios, el rendimiento académico, la capacidad para caer simpático, el compañerismo y la cultura general juegan un rol importante.

El segundo factor, Imagen social, se concentra más bien en los aspectos de status, no menos importantes en toda sociedad, pero particularmente en la peruana, una sociedad dividida por crasas diferencias. Entre los jóvenes, de otro lado, estos factores juegan un rol decisivo en la constitución de su propia autoimagen.

Es explicable la presencia de ambos factores: la adolescencia es una etapa de la vida en la cual la conciencia social y el desarrollo de permanentes interacciones con otras personas adquieren particular significación para la persona y su autoimagen (Davidson \& Neale, 1991).

Los resultados, que son los primeros que se obtienen con esta escala, justifican, como ya se indicó, que esta escala sea empleada en muestras más amplias, con el fin de proporcionar mayor fundamento al reactivo propuesto y explorar sus propiedades en contextos más amplios. 


\section{REFERENCIAS}

Adler, A. (1985). El carácter neurótico. México, DF: Planeta.

Atlas, G. D. (1994). Sensitivity to criticism: a new measure of responses to everyday criticisms. Journal of Psychoeducational Assessment, 12, 241-253.

Auden, W. H. (1996). In defense of gossip. En: Auden, W. H. The complete works of W. H. Auden (vol. 1; ed. por E. Mendelson). Londres: Faber \& Faber, 425-430.

Baron, R. A. (1988). Negative effects of destructive criticism: impact on conflict, self-efficacy and task performance. Journal of Applied Psychology, 73, 199-207.

Beck, A. T., Rush, A. J.; Shaw, B. F. \& Emery, G. (1979). Cognitive theory of depression. Nueva York: Guilford Press.

Berrios, G. (2000). De la fenomenología a la estadística. En: Bulbena Vilarrasa, A.; Berrios, G. \& Fernández de Larrinoa Palacios, P. (Eds.), Medición clínica en psiquiatría y psicología. Barcelona: Masson, 3-13.

Boring, E. G. (1978). The psychology of controversy. En: Hilgard, E. R. (Ed.). American psychology in historical perspective. Addresses of the presidents of the American Psychological Association. Washington DC: American Psychological Association, 233250.

Boyce, P., Hickie, I.; Parker, G. \& Mitchell, P. (1993). Specificity of interpersonal sensitivity to non-melancholic depression. Journal of Affective Disorders, 27, 101-105.
Boyce, P. \& Parker, G. (1989). Development of a scale to measure interpersonal sensitivity. Australian and New Zealand Journal of Psychiatry, 23, 341351.

Buck, R. (1975). Nonverbal communication of affect in children. Journal of Personality and Social Psychology, 31, 644-653.

Caputi, J. (1993). Gossips, Gorgons and Crones. The fates of the earth. Santa Fe, New Mexico: Bear and Company.

Claridge, G. S. \& Broks, P. (1984). Schizotypy and hemispheric function. I. Theoretical considerations and the measurement of schizotypy. Personality and Individual Differences, 5, 633-648.

Davidson, G. G. \& Neale, J. M. (1991). Psicología de la conducta anormal. Enfoque clínico experimental. México, DF: Limusa.

Davis, M. (1980). A multidimensional approach to individual differences in empathy. JSAS: Catalog of Selected Documents in Psychology, 10, 85.

Davis, M. (1983). Measuring individual differences in empathy: evidence for a multidimensional approach. Journal of Personality and Social Psychology, 13, 127-133.

Ebert, M. H.; Loosen, P. T. \& Nurcombe, B. (Eds.) (2000). Current diagnosis \& treatment in psychiatry. Nueva York: Lange Medical Books.

Ekman, P. (1973). Cross cultural studies of facial expression. En: Ekman, P., (Ed.). Darwin and facial expression. San Diego: Academic Press, 169-222.

Fone, B. (2000). Homophobia. A history. Nueva York: Metropolitan Books. 
Forrester, J. (1995). Seducciones del psicoanálisis. Freud, Lacan y Derrida. México, DF: Fondo de Cultura Económica.

Gluckman, M. (1963). Gossip and scandal. Current Anthropology, 4, 307-316.

Goodman, R. F. y Ben-Ze'ev, A. (Eds.) (1994). Good gossip. Lawrence: University Press of Kansas.

Grunfeld, F. V. (1980). Profetas malditos. El mundo trágico de Freud, Einstein, Mahler y Kafka. Barcelona: Planeta.

Hall, J. A. (1984). Nonverbal sex differences: communication accuracy and expressive style. Baltimore: Johns Hopkins University.

Hall, R. \& Goldberg, D. (1977). The role of social anxiety in social interaction difficulties. British Journal of Psychiatry, 131, 610-615.

Handlbauer, B. (1990). Die Adler-FreudKontroverse. Francfort: Fischer.

Harb, G. C.; Heimberg, R. G.; Fresco, D. M.; Schneier, F. R. \& Liebowitz, M. R. (2002). The psychometric properties of the Interpersonal Sensitivity Measure in social anxiety disorder. Behaviour Research and Therapy, 40, 961-979.

Hooley, J. M. \& Teasdale, J. D. (1989). Predictors of relapse in unipolar depressives: expressed emotion, marital distress, and perceived criticism. Journal of Abnormal Psychology, 98, 229-235.

Horrocks, J. E. (1984). Psicología de la adolescencia. México, DF.: Trillas.

Ilgen, D. R.; Peterson, R. B.; Martin, B. A. \& Boeschen, D. A. (1981). Supervisor and subordinate reactions to perfomance appraisal sessions. Organizational
Behavior and Human Perfomance, 30, 15-40.

Kretschmer, E. (1966). Der sensitive Beziehungswahn. Ein Beitrag zur Paranoiafrage und zur psychiatrischen Charakterlehre. Heidelberg: Springer.

Johnson, P. (2000). Intelectuales. Barcelona: Vergara.

Larson, J. R., Jr. (1984). The performance feedback process: a preliminary model. Organizational Behavior and Human Performance, 33, 42-76.

Larson, J. R., Jr. (1986). Supervisors's performance feedback to subordinates: the role of subordinate performance valence and outcome dependence. Organizational Behavior and Human Decision Process, 37, 391-408.

Levin, J. y Arluke, A. (1987). Gossip. The inside scoop. Nueva York: Plenum Press.

Lucock, M. P. \& Salkovskis, P. M. (1988). Cognitive factors in social anxiety and its treatment. Behavior Research and Therapy, 26, 297-302.

Medini, G. y Rosenberg, E. H. (1976). Gossip and psychotherapy. American Journal of Psychotherapy, 30, 452-462.

Mehrabian, A. (1976). Questionnaire measures of affiliative tendency and sensitivity to rejection. Psychological Reports, 38, 199-209.

Millon, Th. (2000). Trastornos de la personalidad. Más allá del DSM-IV. Barcelona: Masson.

Niven, D. (2003). Los 100 secretos de la gente exitosa. Lo que los científicos han descubierto y cómo puede aplicarlo a su vida. Bogotá: Norma. 
O'Neill, E. (1960). Antes del desayuno. En: O'Neill, E. Viaje a la noche y otros ocho dramas. Buenos Aires: Sudamericana. Vol. 2, 175-188.

Ortega, J. (1978). La cultura peruana. Experiencia y conciencia. México, DF: Fondo de Cultura Económica.

Paz, O. (1990). El laberinto de la soledad. México, DF: Fondo de Cultura Económica.

Pepper, F. S., (Ed). (1987). The wit and wisdom of the 20th century. A dictionary of quotations. Nueva York: Peter Bedrick Books.

Pérez, F. (1997). La boca. Para bien o para mal. Santo Domingo: Universidad Autónoma de Santo Domingo.

Perry, H. S. (1982). Psychiatrist of America. The life of Harry Stack Sullivan. Cambridge, Mass., y Londres: The Belknap Press of Harvard University Press.

Perugi, G.; Toni, C.; Travierso, M. C.; Akiskal, H. S. (2003). The role of cyclothimia in atypical depresion: toward a data-based reconceptualization of the borderline-bipolar II connection. Journal of Affective Disorders, 73, 87-98.

Schneider, K. (1968). Las personalidades psicopáticas. Madrid: Morata.

Snodgrass, S. E. (1992). Further effects of role versus gender on interpersonal sensitivity. Journal of Personality and Social Psychology, 62, 154-158.

Snyder, C. R.; Smith, T. W.; Angelli, R. W. \& Ingram, R. E. (1985). On the self serving function of social anxiety: shyness as a self-handicapping strategy. Journal of Personality and Social Psychology, 32, 381-390.
Spaks, P. M. (1985). Gossip. Nueva York: Alfred A. Knopf.

Steiner, G. (1995). Después de Babel. Aspectos del lenguaje y la traducción. México, D. F.: Fondo de Cultura Económica.

Stirling, R. B. (1956). Some psychological mechanisms operative in gossip. Social Forces, 34, 262-267.

Sullivan, H. S. (1964). La teoría interpersonal de la psiquiatría. Buenos Aires: Psique.

Veiga, J. F. (1988). Face your problem subordinates now! Academy of Management Executive, 2, 145-152.

Wittig, M. A. \& Skolnick, P. (1978). Status versus warmth as determinants of sex differences in personal space. Sex Roles, 4, 493-503.

Wittkower, R. \& Wittkower, M. (2000). Nacidos bajo el signo de Saturno. Genio y temperamento de los artistas desde la Antigüedad hasta la Revolución Francesa. Madrid: Cátedra.

Zimbardo, Ph. (2001). El niño tímido. Superar y prevenir la timidez desde la infancia. Barcelona: Paidós. 


\section{ANexo \\ Inventario de sensibilidad a la crítica}

Valora cuánto te afectaría una crítica que te formulara tu mejor amigo o amiga acerca de los siguientes aspectos. Emplea para ello la siguiente escala:

$\begin{array}{ccccccccccc}0 & 1 & 2 & 3 & 4 & 5 & 6 & 7 & 8 & 9 & \begin{array}{c}10 \\ \text { Mucho }\end{array}\end{array}$

\begin{tabular}{llllllllllll}
\hline $\mathrm{N}^{\circ} \quad$ Enunciado & 0 & 1 & 2 & 3 & 4 & 5 & 6 & 7 & 8 & 9 & 10 \\
\hline 1. Tu apariencia física & 0 & 1 & 2 & 3 & 4 & 5 & 6 & 7 & 8 & 9 & 10 \\
2. Tu capacidad económica & 0 & 1 & 2 & 3 & 4 & 5 & 6 & 7 & 8 & 9 & 10 \\
3. Tu rendimiento académico & 0 & 1 & 2 & 3 & 4 & 5 & 6 & 7 & 8 & 9 & 10 \\
4. Tu distrito de residencia & 0 & 1 & 2 & 3 & 4 & 5 & 6 & 7 & 8 & 9 & 10 \\
5. Tu universidad & 0 & 1 & 2 & 3 & 4 & 5 & 6 & 7 & 8 & 9 & 10 \\
6. Tus amigos & 0 & 1 & 2 & 3 & 4 & 5 & 6 & 7 & 8 & 9 & 10 \\
7. Tu personalidad & 0 & 1 & 2 & 3 & 4 & 5 & 6 & 7 & 8 & 9 & 10 \\
8. Tu manejo de situaciones & 0 & 1 & 2 & 3 & 4 & 5 & 6 & 7 & 8 & 9 & 10 \\
9. La ropa que usas & 0 & 1 & 2 & 3 & 4 & 5 & 6 & 7 & 8 & 9 & 10 \\
10. Los lugares que frecuentas & 0 & 1 & 2 & 3 & 4 & 5 & 6 & 7 & 8 & 9 & 10 \\
11. La carrera que estudias & 0 & 1 & 2 & 3 & 4 & 5 & 6 & 7 & 8 & 9 & 10 \\
12. El enamorado (o la enamorada) & & & & & & & & & & & \\
$\quad$ que tienes & 0 & 1 & 2 & 3 & 4 & 5 & 6 & 7 & 8 & 9 & 10 \\
13. Tus pasatiempos & 0 & 1 & 2 & 3 & 4 & 5 & 6 & 7 & 8 & 9 & 10 \\
14. Tu capacidad para hacer valer & & & & & & & & & & & \\
tus derechos & 0 & 1 & 2 & 3 & 4 & 5 & 6 & 7 & 8 & 9 & 10 \\
15. Tu rendimiento en algún deporte & 0 & 1 & 2 & 3 & 4 & 5 & 6 & 7 & 8 & 9 & 10 \\
16. Tu capacidad para caer simpático & 0 & 1 & 2 & 3 & 4 & 5 & 6 & 7 & 8 & 9 & 10 \\
17. Tu familia & 0 & 1 & 2 & 3 & 4 & 5 & 6 & 7 & 8 & 9 & 10 \\
18. Tu capacidad para expresarte & 0 & 1 & 2 & 3 & 4 & 5 & 6 & 7 & 8 & 9 & 10 \\
19. Tu sentido de compañerismo & 0 & 1 & 2 & 3 & 4 & 5 & 6 & 7 & 8 & 9 & 10 \\
20. Tu cultura general & 0 & 1 & 2 & 3 & 4 & 5 & 6 & 7 & 8 & 9 & 10 \\
\hline
\end{tabular}

